Check for updates

Cite this: Chem. Commun., 2019, 55, 11025

Received 15th July 2019

Accepted 7th August 2019

DOI: $10.1039 / c 9 c c 05415 d$

rsc.li/chemcomm

\section{A thiazolo[5,4-d]thiazole-bridged porphyrin organic framework as a promising nonlinear optical material $\dagger$}

\author{
Mahalaxmi Samal, ${ }^{a}$ Sreeramulu Valligatla, ${ }^{b}$ Nabil A. Saad, (D) M. Veeramohan Rao, ${ }^{c}$ \\ D. Narayana Rao, (D) ${ }^{c}$ Rojalin Sahu (D)*a and Bishnu P. Biswal (D) *ef
}

\begin{abstract}
Porphyrin-based porous organic frameworks are an important group of materials gaining interest due to their structural diversity and distinct opto-electronic properties. However, these materials are seldom explored for nonlinear optical (NLO) applications. In this work, we investigate a thiazolo[5,4- $d$ ] thiazole-bridged porous, porphyrin framework (Por-TzTz-POF) with promising NLO properties. The planar TzTz moiety coupled with integrated porphyrin units enables efficient $\pi$-conjugation and charge distribution in the Por-TzTz-POF resulting in a high nonlinear absorption coefficient $\left(\beta=1100 \mathrm{~cm} \mathrm{GW}^{-1}\right)$ with figure of merit (FoM) $\sigma_{1} / \sigma_{0}=5571$, in contrast to analogous molecules and material counterparts e.g. metal-organic frameworks (MOFs; $\left.\beta=\sim 0.3-0.5 \mathrm{~cm} \mathrm{GW}^{-1}\right)$, molecular porphyrins $(\beta=\sim 100-$ $\left.400 \mathrm{~cm} \mathrm{GW}^{-1}\right)$, graphene $\left(\beta=900 \mathrm{~cm} \mathrm{GW}^{-1}\right)$, and covalent organic frameworks (Por-COF-HH; $\beta=1040 \mathrm{~cm} \mathrm{GW}^{-1}$ and FoM = 3534).
\end{abstract}

In the realm of nonlinear optics (NLO), molecular porphyrins have unique prominence due to their outstanding optoelectronic properties, large and fast NLO responses, possibility of engineering with metals, and excellent thermo-chemical stabilities, making them suitable for applications in telecommunication, data storage, sensors, and display technologies. ${ }^{1}$ However, there are still enough opportunities to fine-tune the optical nonlinearities of singular molecular porphyrins, which can be achieved by de novo design of integrated porphyrin units with extended $\pi$-conjugations and periodic incorporation of opto-electronically active heterocyclic units. ${ }^{2}$ The large $\pi$-electron

\footnotetext{
${ }^{a}$ Department of Chemistry, School of Applied Sciences, Kalinga Institute of Industrial Technology (KIIT), Deemed to be University, Bhubaneswar-24, Odisha, India. E-mail: rsahufch@kiit.ac.in; Fax: +91-674 2725113; Tel: +91-9778127994 ${ }^{b}$ Institute for Integrative Nanosciences, IFW-Dresden, 01069, Dresden, Germany

${ }^{c}$ School of Physics, University of Hyderabad, Hyderabad-500046, India

${ }^{d}$ Department of Physics, Pondicherry University, Pondicherry-606015, India

${ }^{e}$ Faculty of Chemistry and Food Chemistry, Center for Advancing Electronics Dresden, Technische Universität Dresden, 01062, Dresden, Germany

${ }^{f}$ Max-Planck-Institute for Solid State Research, Heisenbergstraße 1, 70569 Stuttgart, Germany. E-mail: b.biswal@fkf.mpg.de

$\dagger$ Electronic supplementary information (ESI) available: Detailed experimental data and characterization. See DOI: 10.1039/c9cc05415d
}

delocalization in heterocycles would offer efficient light absorbability and thus red shifting of their absorption bands along with in-plane facile charge transport, which is crucial for optoelectronic applications like NLO. ${ }^{3,4}$ There are few reports on linear conjugated porphyrin oligomers, which have shown excellent NLO response. However, arduous multistep synthesis appears to be the bottleneck toward their development. ${ }^{5}$

Conjugated, porous organic frameworks comprising of porphyrin units (POFs) ${ }^{6}$ are of high interest due to their scalable protocols, in addition to excellent chemical stability, diverse functionality, and unique opto-electronic properties. Despite this, few have reported successful introduction of heterocyclic units into porous networks. ${ }^{7}$ Introduction of fused (bi)heterocyclic units e.g. thiazolo[5,4- $d]$ thiazole (TzTz) into a porous polymeric network is interesting because of their semiconductor characteristics associated with the electron deficient nature, high oxidative stability, and a rigid planar structure. ${ }^{8}$ Notably, TzTz moieties feature an excellent photoabsorbing ability ${ }^{9}$ and strong electron acceptor characteristics; when linked with porphyrin units, they are expected to be highly useful in photo(electro)catalysis and opto-electronics applications..$^{10}$ Until recently, none of the POFs or COFs composed of porphyrin units have been explored as NLO materials. We recently reported three regioregular crystalline Por-COFs ${ }^{11}$ with outstanding NLO activity, which inspired us to further investigate and understand analogous porous materials for advanced NLO applications.

Herein, we present a new conjugated, porous TzTz-linked porphyrin organic framework (Por-TzTz-POF) by single step condensation of 5,10,15,20-tetrakis(4-formylphenyl)-21 H,23Hporphyrin (TFPP) and dithiooximide (DTO) (Fig. 1). The PorTzTz-POF is porous with a Brunauer-Emmett-Teller (BET) surface area of $740 \mathrm{~m}^{2} \mathrm{~g}^{-1}$, which is higher than those of most of the TzTz-linked frameworks reported so far in the literature. ${ }^{12} \mathrm{We}$ also examined the NLO activity of this Por-TzTz-POF by the nanosecond laser open aperture Z-scan technique under $532 \mathrm{~nm}$ excitation. ${ }^{13}$ Interestingly, the Por-TzTz-POF shows optical switching behavior from saturable absorption (SA) to reverse saturable absorption (RSA) with increased input laser intensity, 
a)

and a promising nonlinear absorption coefficient value $(\beta=$ $\left.\sim 1100 \mathrm{~cm} \mathrm{GW}^{-1} ; \sigma_{1} / \sigma_{0}(\mathrm{FoM})=5571\right)$ compared to our previously reported metal-free imine-linked porphyrin-COF (Por-COF-HH), ${ }^{11}$ $\beta=1040 \mathrm{~cm} \mathrm{GW}^{-1}$ and $\sigma_{1} / \sigma_{0}(\mathrm{FoM})=3534$. This result reveals the prevalence of (bi)heterocycle-bridged porphyrin frameworks for optical switching NLO applications.

The Por-TzTz-POF was synthesised using the standard solvothermal protocol as described in Section S2, ESI. $\dagger$ To carve out the structure-activity relationship in the porphyrin frameworks, and for comparison, we have synthesized a reported crystalline, Schiff-base analogous Por- $\mathrm{C}=\mathrm{N}-\mathrm{COF}$ by condensation of TFPP and 5,10,15,20-tetrakis(4-aminophenyl)-21 H,23Hporphyrin (TAPP) (Fig. 1a). The synthesized porphyrin frameworks were examined via powder X-ray diffraction (PXRD) to investigate their crystallinity and phase purity. From PXRD, it is confirmed that the Por-TzTz-POF is amorphous in nature with a broad reflection centred at $18^{\circ} 2 \theta$ (Fig. 1b). However, the Por$\mathrm{C}=\mathrm{N}-\mathrm{COF}$ is crystalline with the most intense first peak at a low $2 \theta$ angle of $\sim 4.7^{\circ}$ corresponding to the (100) reflection, along with other minor peaks (Fig. 1c). To gain further insight into the functional groups and the local bonding modes in the Por-TzTz-POF, we recorded the Fourier Transform Infrared (FT-IR) spectrum, from which, the newly formed heterocyclic $\mathrm{C}=\mathrm{C}$ bond $\left(1736 \mathrm{~cm}^{-1}\right)$ and $\mathrm{C}=\mathrm{N}$ bond $\left(1594 \mathrm{~cm}^{-1}\right)$ can be identified. The near disappearance of $\mathrm{C}=\mathrm{O}$ stretching vibration of the carbonyl group (expected range $1693 \mathrm{~cm}^{-1}$ ) confirms the condensation of the formyl group of TFPP and DTO to form the (bi)heterocyclic TzTz linkages. Furthermore, the FT-IR spectrum shows minor bands corresponding to the $-\mathrm{C}=\mathrm{N}$ and $-\mathrm{C}-\mathrm{S}$ bonds of the TzTz ring (Fig. S3, ESI $\dagger$ ). The framework structure and composition of the Por-TzTz-POF were confirmed by ${ }^{13} \mathrm{C}$ cross-polarization magic-angle spinning (CP-MAS) NMR spectroscopy (Fig. 2a). All distinct signals can be assigned to the respective carbon atoms of the porphyrin framework. The spectrum of the Por-TzTz-POF shows the presence of aromatic groups (a chemical shift region of $c a$. 110-150 ppm) as well as higher chemical shifts for the carbons of the (bi)heterocyclic TzTz ring, $\delta=\sim 170 \mathrm{ppm}(\mathrm{S}-\mathrm{C}=\mathrm{N})$ and $152 \mathrm{ppm}(\mathrm{C}=\mathrm{C})$.

The thermal stability of the Por-TzTz-POF was verified using thermogravimetric analysis (TGA) under an $\mathrm{N}_{2}$ atmosphere. Mass loss shows that about $7 \%$ of residual solvent molecules were trapped inside the Por-TzTz-POF, which were removed upon gradual heating to $\sim 150{ }^{\circ} \mathrm{C}$. Overall, the framework was stable up to $\sim 470{ }^{\circ} \mathrm{C}$. After decomposition, a weight loss of $\sim 50 \%$ for the Por-TzTz-POF was observed until the end of the scan (Fig. S4, ESI $\dagger$ ). $\mathrm{N}_{2}$ adsorption/desorption analysis of the Por-TzTz-POF displays a typical reversible type-II isotherm with a rapid uptake at low relative pressures giving rise to a BET surface area of $740 \mathrm{~m}^{2} \mathrm{~g}^{-1}$ (Fig. 2b). This value is comparatively higher than those of most of the TzTz-linked POFs reported in the literature such as $604 \mathrm{~m}^{2} \mathrm{~g}^{-1}$ (TzTz-POP-3), $491 \mathrm{~m}^{2} \mathrm{~g}^{-1}$ (TzTz-POP-5), $299 \mathrm{~m}^{2} \mathrm{~g}^{-1}$ (TzTz-POP-1) and $488 \mathrm{~m}^{2} \mathrm{~g}^{-1}$ (TzTzPOP-2). ${ }^{12}$ The pore size distribution (PSD) was derived from the $\mathrm{N}_{2}$ adsorption data for the Por-TzTz-POF using nonlocal density functional theory (NLDFT), which suggests narrow micropores of these polymeric frameworks with a major pore diameter of $1.3 \mathrm{~nm}$. This is slightly smaller than the pore diameter value obtained $(1.6 \mathrm{~nm})$ from the ideal structure model (Fig. S5, ESI $\dagger$ ). The external morphology of the Por-TzTz-POF was visualized by scanning electron microscopy (SEM), which revealed small particles $(\sim 30-50 \mathrm{~nm})$, with cubic morphology, for large agglomerations (Fig. S6a and b, ESI $\dagger$ ). A similar morphology was also observed in transmission electron microscopy (TEM) images (Fig. 2d and Fig. S6c and d, ESI $\dagger$ ). It is worth 
a)

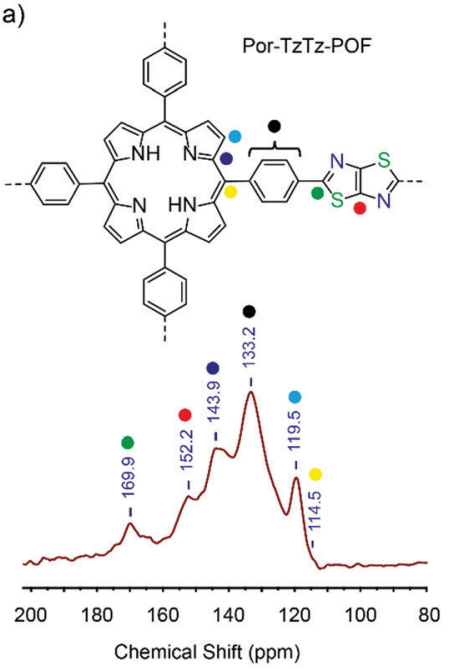

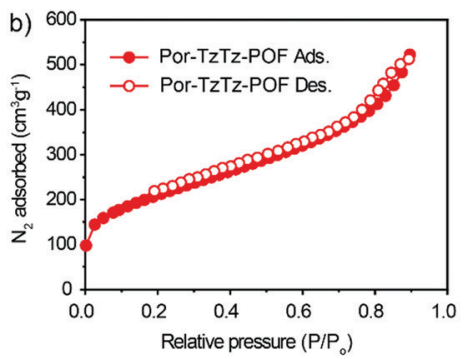
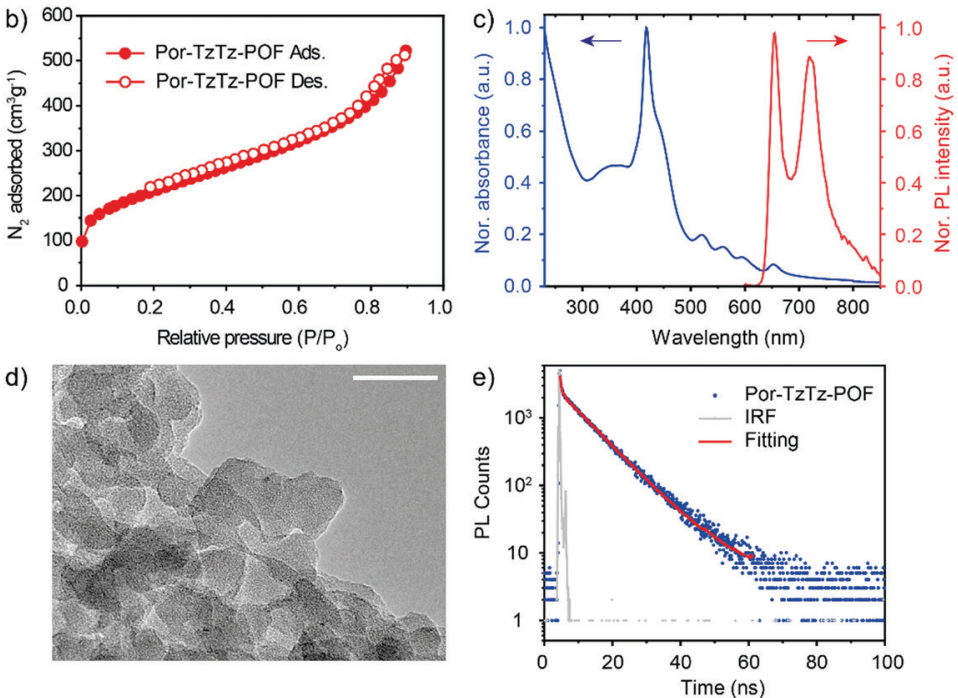

Fig. 2 (a) ${ }^{13} \mathrm{C}$ cross-polarization magic-angle spinning (CP-MAS) solid state NMR spectra of the Por-TzTz-POF; (b) $\mathrm{N}_{2}$ physisorption isotherms collected at $77 \mathrm{~K}$; (c) UV-vis and photoluminescence spectrum (measured in the integration sphere in the dispersion state in 2-propanol); (d) TEM images of the Por-TzTz-POF (scale bar $=50 \mathrm{~nm}$ ); and (e) time-correlated single-photon counting experiment for the Por-TzTz-POF measured in 2-propanol. The sample was excited with a $\lambda_{\mathrm{ex}}=380 \mathrm{~nm}$ laser and emission was measured at $\lambda_{\mathrm{em}}=655 \mathrm{~nm}$.

mentioning that the particle size and shape of any organic porous materials synthesized via the solvothermal method are hard to predict and drastically vary due to a range of interactions such as $\pi-\pi$ stacking, van der Waals interactions etc.

The optoelectronic properties such as UV-vis absorption and photoluminescence (PL) measurements of the Por-TzTz-POF were accessed using the center mount position of an integrating sphere in the dispersion state (2-propanol). The UV-vis spectral studies display all four Q-band components [500-700 nm: $\left.\mathrm{Q}_{y}(1,0), \mathrm{Q}_{y}(0,0), \mathrm{Q}_{x}(1,0), \mathrm{Q}_{x}(0,0)\right]$ for the Por-TzTz-POF (Fig. 2c). The Soret $\mathrm{B}$-band $(\mathrm{B}(0,0))$ is associated with a single component of $418 \mathrm{~nm}$ (dominant major peak) similar to the reported Por$\mathrm{C}=\mathrm{N}$-COF. ${ }^{11}$ However, an additional shoulder peak appears at $442 \mathrm{~nm}$ for the Por-TzTz-POF in contrast to the Por-C $=\mathrm{N}-\mathrm{COF}$, which corresponds to the TzTz group. We observed an emission at $655 \mathrm{~nm}$ in the PL analysis (Fig. 2c), which is identical to that of the reported Por- $\mathrm{C}=\mathrm{N}-\mathrm{COF}$ compound. The fluorescence decays can be fitted with (bi)exponential functions (Fig. 2e) and the amplitude weighted average lifetime for the Por-TzTzPOF is $7.5 \mathrm{~ns}\left[\tau_{1}=0.41 \mathrm{~ns}(12.2 \%)\right.$ and $\left.\tau_{2}=8.46 \mathrm{~ns}(87.8 \%)\right]$, which is lower than that of the Por-C $=\mathrm{N}-\mathrm{COF}\left(\tau_{\text {avg }}=9.17 \mathrm{~ns}\right)$.

In light of the exciting opto-electronic features of the PorTzTz-POF, the NLO response was examined by the open aperture $Z$-scan technique at an excitation wavelength of $532 \mathrm{~nm}$ from a frequency doubled Nd:YAG laser with varying input intensities. A purely intensity dependent, nonlinear absorption mechanism was observed from the $Z$-scan profiles at different input intensities (Fig. 3a). For comparison, the NLO response of Por- $\mathrm{C}=\mathrm{N}-\mathrm{COF}^{11}$ is also presented in Fig. 3b. Interestingly, it has been found that both the porphyrin frameworks exhibit NLO switching behavior as the nonlinear absorption swap from saturable absorption (SA) to reverse saturable absorption (RSA) with increasing input intensity. Fig. 3 denotes the open aperture $Z$-scan curves for the (a) Por-TzTz-POF and (b) Por-C $=\mathrm{N}-\mathrm{COF}$ at

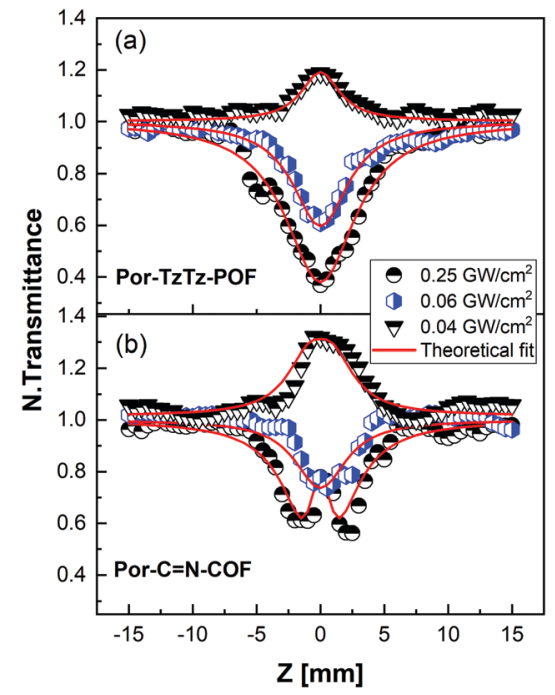

Fig. 3 Open aperture Z-scan traces for (a) Por-TzTz-POF and (b) Por$\mathrm{C}=\mathrm{N}-\mathrm{COF}^{11}$ under the excitation at $532 \mathrm{~nm}$, ns laser at different input intensities. The solid lines represent the theoretical fitting, and triangles, spheres and hexagons represent the experimental data.

peak intensities of $0.04,0.06$ and $0.25 \mathrm{GW} \mathrm{cm}{ }^{-2}$. At a lower intensity of $0.04 \mathrm{GW} \mathrm{cm}^{-2}$, both materials showed SA behavior. As the input intensity increases, the excited state absorption leads to characteristic RSA behaviour as depicted in Fig. 3. However, in the case of the Por- $\mathrm{C}=\mathrm{N}-\mathrm{COF}$, at an input intensity of $0.25 \mathrm{GW} \mathrm{cm}^{-2}$, an optical switching behavior switch from RSA to SA is observed and is attributed to the dominant excited state absorption at lower intensities (far-focus) and saturation of excited states at higher intensity (near-focus). To further support this hypothesis, the known theoretical model was applied with five level rate equations (Section S5, ESI $\dagger$ ). ${ }^{14}$ It has been found 
that the experimental results are in good agreement with the theoretical fits at different input intensities (Fig. 3). From the fitting results, the nonlinear absorption coefficient $(\beta)$, ground state absorption cross section $\left(\sigma_{0}\right)$, first excited state $\left(\sigma_{1}\right)$ and second excited state absorption cross sections $\left(\sigma_{2}\right)$ have been determined for both Por-TzTz-POF and Por-C $=\mathrm{N}-\mathrm{COF}$. A slightly higher $\beta$ value is observed for the Por-TzTz-POF $(\beta=$ $\left.1100 \mathrm{~cm} \mathrm{GW}^{-1}\right)$ compared to the Por- $\mathrm{C}=\mathrm{N}-\mathrm{COF}\left(\beta=1040 \mathrm{~cm} \mathrm{GW}^{-1}\right)$ (Table S1, ESI $\dagger$ ), and the value is much higher than those of many other reported materials such as metalated porphyrins $\left(\beta=132-366 \mathrm{~cm} \mathrm{GW}^{-1}\right)$, MOFs $\left(\beta=0.28-0.46 \mathrm{~cm} \mathrm{GW}^{-1}\right)$ and graphene $\left(\beta=900 \mathrm{~cm} \mathrm{GW}^{-1}\right) .{ }^{11,14,15}$ We assume that the higher NLO response of the Por-TzTz-POF could be attributed to a combined effect of the structural planarity of the TzTz-phenyl core (quasi-planar: dihedral angle $6.9^{\circ}$ ), effective inter-layer stacking, efficient $\pi$-conjugation-electron transport and framework disorderliness (loss of symmetry) compared to the imine $(\mathrm{C}=\mathrm{N})$-phenyl core (dihedral angle $\left.62.8^{\circ}\right)$ in the case of the Por$\mathrm{C}=\mathrm{N}-\mathrm{COF}$ (Fig. S9, ESI $\dagger$ ). ${ }^{12}$ Moreover, the obtained figure of merit (FoM $=\sigma_{1} / \sigma_{0} ; 5571$ ) for the Por-TzTz-POF is also higher than that of our recently reported porphyrin based COFs; PorCOF-HH $($ FoM = 3534), Por-COF-ZnCu (FoM = 3565) and PorCOF-ZnNi (FoM = 3762), tabulated in Table S2 (ESI $\dagger) .{ }^{11}$ Overall, these results signify a structure-activity relationship in porphyrin based porous organic materials that can be useful in optical switching applications. ${ }^{16,17}$

In summary, we have synthesized a conjugated TzTz-linked porphyrin organic framework employing single-step direct condensation of A4 porphyrin tetra-aldehyde and DTO. Moreover, to prove the concept and to draw the benefits from infinite conjugated porphyrin networks, the NLO response of the PorTzTz-POF has been investigated using the $Z$-scan technique. Next, the NLO activity of the Por-TzTz-POF was compared with that of a reported analogous crystalline Schiff base Por-COF (Por-C $=\mathrm{N}-\mathrm{COF}$ ). Although, optical switching behavior from SA to RSA with input intensity was observed for both materials, the amorphous Por-TzTz-POF is found to display higher nonlinear absorption coefficient values $\left(\beta=\sim 1100 \mathrm{~cm} \mathrm{GW}^{-1}\right.$ and FoM $=$ 5571). Through this work, we tried to establish a structureactivity relationship in porphyrin containing organic frameworks. However, there is still a huge opportunity to further explore these materials at the molecular level, to help develop them for next generation NLO applications.

B. P. B. acknowledges the Alexander von Humboldt foundation for a research fellowship (Award reference number: Ref 3.5IND-1188443-HFST-P). We are thankful to Prof. Xinliang Feng for the research support and helpful discussion. We thank Prof. Ulrich Starke and Dr Kathrin Müller for XPS measurements. Open Access funding provided by the Max Planck Society.

\section{Conflicts of interest}

The authors declare no competing financial interest.

\section{Notes and references}

1 (a) P. N. Prasad and D. J. Williams, Introduction to Nonlinear Optical Effects in Molecules and Polymers, Wiley, New York, USA, 1990; (b) G. D. L. Torre, P. Vazquez, F. Agullo-Lopez and T. Torres, Chem. Rev., 2004, 104, 3723-3750.

2 (a) Y.-L. Liu, Z.-B. Liu, J.-G. Tian, Y. Zhu and J.-Y. Zheng, Opt. Commun., 2008, 281, 776-781; (b) W. J. Su, T. M. Cooper and M. C. Brant, Chem. Mater., 1998, 1212-1213.

3 (a) A. Krivocapic, H. L. Anderson, G. Bourhill, R. Ives, S. Clark and K. J. McEwan, Adv. Mater., 2001, 13, 652-656; (b) B. A. Tsuda and A. Osuka, Science, 2001, 293, 79-82.

4 I.-W. Hwang, T. Kamada, T. K. Ahn, D. M. Ko, T. Nakamura, A. Tsuda, A. Osuka and D. Kim, J. Am. Chem. Soc., 2004, 126, 16187-16198.

5 (a) N. Aratani and A. Osuka, Chem. Rec., 2003, 3, 225-234; (b) M. Terazima, H. Shimizu and A. Osuka, J. Appl. Phys., 1997, 81, 2946-2951.

6 (a) R. Shen, W. Zhu, X. Yan, T. Li, Y. Liu, Y. Li, S. Daia and Z.-G. Gu, Chem. Commun., 2019, 55, 822-825; (b) R. Chen, J. L. Shi, Y. Ma, G. Lin, X. Lang and C. Wang, Angew. Chem., Int. Ed., 2019, 58, 6430-6433; (c) H. Liao, H. Wang, H. Ding, X. Meng, H. Xu, B. Wang, X. Ai and C. Wang, J. Mater. Chem. A, 2016, 4, 7416-7421; (d) B.-Q. Li, S.-Y. Zhang, X. Chen, C.-Y. Chen, Z.-J. Xia and Q. Zhang, Adv. Funct. Mater., 2019, 29, 1901301; (e) B.-Q. Li, S.-Y. Zhang, B. Wang, Z.-J. Xia, C. Tanga and Q. Zhang, Energy Environ. Sci., 2018, 11, 1723-1729; $(f)$ Z.-J. Xia, H.-C. Yang, Z. Chen, R. Z. Waldman, Y. Zhao, C. Zhang, S. N. Patel and S. B. Darling, Adv. Mater. Interfaces, 2019, 1900254.

7 J. Y. Jung, M. Kang, J. Chun, J. Lee, J. Kim, J. Kim, Y. Kim, S. J. Kim, C. Lee and J. Yoon, Chem. Commun., 2013, 49, 176-178.

8 A. Dessì, M. Calamante, A. Mordini, M. Peruzzini, A. Sinicropi, R. Basosi, F. Fabrizi de Biani, M. Taddei, D. Colonna, A. di Carlo, G. Reginato and L. Zani, RSC Adv., 2015, 5(41), 32657-32668.

9 B. P. Biswal, H. A. Vignolo-González, T. Banerjee, L. Grunenberg, K. Gottschling, J. Nuss and B. V. Lotsch, J. Am. Chem. Soc., 2019, 141(28), 11082-11092.

10 A. N. Woodward, J. M. Kolesar, S. R. Hall, N. A. Saleh, D. S. Jones and M. G. Walter, J. Am. Chem. Soc., 2017, 139(25), 8467-8473.

11 B. P. Biswal, S. Valligatla, M. Wang, T. Banerjee, N. Momen, B. M. K. Mariserla, N. Chandrasekhar, D. Becker, M. Addicoat, I. Senkovska, R. Berger, D. N. Rao, S. Kaskel and X. Feng, Angew. Chem., Int. Ed., 2019, 58, 6896-6900.

12 (a) B. P. Biswal, D. Becker, N. Chandrasekhar, J. S. Seenath, S. Paasch, S. Machill, F. Hennersdorf, E. Brunner, J. J. Weigand, R. Berger and X. Feng, Chem. - Eur. J., 2018, 24, 10868-10875; (b) X. Zhu, C. Tian, T. Jin, J. Wang, S. M. Mahurin, W. Mei, Y. Xiong, J. Hu, X. Feng, H. Liu and S. Dai, Chem. Commun., 2014, 50, 15055-15058.

13 M. O. Senge, M. Fazekas, E. G. A. Notaras, W. J. Blau, M. Zawadzka, O. B. Locos and E. M. N. Mhuircheartaigh, Adv. Mater., 2007, 19, $2737-2774$

14 (a) M. B. M. Krishna, V. P. Kumar, N. Venkatramaiah, R. Venkatesan and D. N. Rao, Appl. Phys. Lett., 2011, 98, 0811061; (b) M. SheikBahae, A. A. Said, T. Wei, D. J. Hagan and E. W. Van Stryland, IEEE J. Quantum Electron., 1990, 26, 760-769; (c) V. Sreeramulu, K. K. Haldar, A. Patra and D. N. Rao, Opt. Laser Technol., 2016, 118, 30333-30341; (d) M. B. M. Krishna, N. Venkatramaiah and D. Narayana Rao, J. Opt., 2014, 16, 015205.

15 (a) J. Li, D. Jia, S. Meng, J. Zhang, M. P. Cifuentes, M. G. Humphrey and C. Zhang, Chem. - Eur. J., 2015, 21, 7914-7926; (b) X. Jiang, L. Zhang, S. Liu, Y. Zhang, Z. He, W. Li, F. Zhang, Y. Shi, W. Lü, Y. Li, Q. Wen, J. Li, J. Feng, S. Ruan, Y.-J. Zeng, X. Zhu, Y. Lu and H. Zhang, Adv. Opt. Mater., 2018, 1800561; (c) X. Li, Q. Gao, J. Aneesh, H.-S. Xu, Z. Chen, W. Tang, C. Liu, X. Shi, K. V. Adarsh, Y. Lu and K. P. Loh, Chem. Mater., 2018, 30, 5743-5749; (d) M. B. M. Krishna, N. Venkatramaiah, R. Venkatesan and D. N. Rao, J. Mater. Chem., 2012, 22, 3059-3068.

16 F. Castet, V. Rodriguez, J.-L. Pozzo, L. Ducasse, A. Plaquet and B. Champagne, Acc. Chem. Res., 2013, 46, 2656-2665.

17 V. Sreeramulu, K. K. Haldar, A. Patra and D. N. Rao, J. Phys. Chem. C, 2014, 118, 30333-30341. 